\title{
Transients in ecology: stochasticity, management, and understanding
}

\author{
Karen C. Abbott ${ }^{1} \cdot \operatorname{Kim}$ Cuddington ${ }^{2} \cdot$ Alan Hastings ${ }^{3,4}$ \\ Published online: 8 December 2021 \\ (c) The Author(s), under exclusive licence to Springer Nature B.V. 2021
}

The history of theoretical ecology is replete with insights earned by studying the long-term, equilibrium dynamics that result from processes playing out within populations and communities. However, real systems take time to reach equilibrium, and some may never reach it due to the influence of stochasticity and disturbance. It is therefore increasingly apparent that by focusing on equilibria, we miss many important aspects of the dynamics we can expect to see on ecologically relevant time scales. Overviews of these issues have been presented in a series of papers by the three coeditors of this special feature along with our collaborators (Hastings et al. 2018, 2021; Morozov et al. 2020; Francis et al. 2021). The focus of this previous work has been on big picture synthesis, at the expense of detailed studies of particular systems or issues. The papers in this special feature fill that gap admirably and provide new and important insights on the influence of stochasticity and implications for both basic understanding and management.

In the particular case of disease, the importance of transient dynamics is painfully and clearly illustrated by the current global pandemic, where transient COVID-19 outbreaks are a far greater concern than the eventual equilibrium. From this compelling starting point, Tao, Hite, Lafferty, Earn, and Bharti present a wide-ranging survey of where, when, and why understanding transient dynamics is crucial for evaluating and managing human and wildlife disease (Tao et al. 2021). They illustrate through examples how transient effects at one scale - considering temporal and spatial scales, as

Alan Hastings

amhastings@ucdavis.edu

1 Department of Biology, Case Western Reserve University, Cleveland, OH 44106, USA

2 Department of Biology, University of Waterloo, Waterloo, ON N2L 3G1, Canada

3 Department of Environmental Science and Policy, University of California, Davis, CA 95616, USA

4 Santa Fe Institute, 1399 Hyde Park Road, Santa Fe, NM 87501, USA well as levels of organization from individual hosts to populations and communities - influence transients at other scales. For instance, a transient physiological response to infection in an individual host and transient host movement (and thus contact) patterns interact with transient population and community dynamics (which influence density dependent disease transmission) to determine the overall trajectory of a disease. Transients at different scales may have different characteristic lifetimes, creating a rich and complex set of possible outcomes. Tao and co-authors powerfully illustrate the role of theory in understanding such outcomes and point to many exciting avenues for future research.

With their focus on species extinction, Vidiella, Fontich, Valverde, and Sardanyés address another crucially important application for ecological theory (Vidiella et al. 2021). It is well known that when changing conditions (e.g., global change) cause extinctions, species do not go extinct instantaneously but rather approach this new zero equilibrium over some amount of time. Persistence is then a transient state, and understanding the duration of this transient is key to remediation. Vidiella and co-authors study communities of one to three species and characterize different routes to extinction with respect to their associated transient dynamics. Their work emphasizes quite elegantly how an understanding of the cause of transient dynamics leads directly to improved predictions about how quickly a species may go extinct following different types of stressors (e.g., facilitation breakdown versus habitat loss). This knowledge is critical, as it establishes the necessary timescales for any protective actions, if they are to succeed.

Boettiger likewise addresses the question of managing ecological systems with long transients (Boettiger 2021) but notes that, given the ubiquitous presence of stochasticity, it may not be possible to know whether a system is currently in a transient state or at equilibrium. He compares a system with alternative stable states, currently in a (desirable) low stable state and at risk of stochastically switching to the (undesirable) high stable state, to a system that is currently in a low transient state and expected to eventually approach a high equilibrium state. Given unavoidable uncertainty about 
the nature of the current state (equilibrial versus transient), how best to manage the system? Boettiger's analysis not only illustrates how management can be misled if transients are misidentified as equilibria, but also shows how dynamic and adaptive management strategies allow managers to discover successful actions even with imperfect knowledge.

The impact of stochasticity on transient dynamics is further explored in the remaining two papers in this special feature. In their work, Reimer, Arroyo-Esquivel, Jiang, Scharf, Wolkovich, Zhu, and Boettiger assess the performance of different statistical methods in identifying the "true" dynamics underlying noisy simulated time series (Reimer et al. 2021). As expected, their results confirm the difficulty of identifying transient states in time series data, particularly when presented with just one or a small number of realizations. Nevertheless, their work reveals intriguing features of each analysis, such as the ability of hidden Markov models to identify distinct states within a time series and the complementary insights provided by parametric and nonparametric model fits. This thought-provoking work challenges readers to consider the impacts of stochasticity on transient dynamics and more carefully examine both the virtues and limitations of time series analysis in light of transients.

Boettiger and Reimer et al. both consider systems that have a long-lived transient state and a stable state and note the resemblance to systems with alternative stable states. Abbott and Dakos explore this resemblance in depth, focusing on the response of each type of system to different levels of stochasticity (Abbott and Dakos 2021). They find that either scenario - alternative stable states or one stable state and a long transient - generates a very similar bimodal distribution of states through time, observed in a very similar intermediate-intensity noise range. They use this finding to suggest that transient dynamics are likely to be mistaken for equilibrium states. Returning to the broader theme of this special feature, their work argues for the importance of considering transient dynamics across ecology: transients may not reveal themselves if we fail to look for them, and if we tacitly assume equilibrium dynamics, we will not understand what we are missing.

Acknowledgements The co-editors of the special feature, and many of the authors of the papers within it, participated in the Transients in Biological Systems Investigative Workshop at the National Institute for Mathematical and Biological Synthesis (NIMBioS), supported by the National Science Foundation through NSF Award \#DBI-1300426, with additional support from The University of Tennessee, Knoxville. We gratefully acknowledge NIMBioS for promoting the intellectual exchange that lead to this feature.

\section{References}

Abbott KC, Dakos V (2021) Mapping the distinct origins of bimodality in a classic model with alternative stable states. Theor Ecol this issue

Boettiger C (2021) Ecological management of stochastic systems with long transients. Theor Ecol this issue

Francis TB, Abbott KC, Cuddington K, Gellner G, Hastings A, Lai Y-C, Morozov A, Petrovskii S, Zeeman ML (2021) Management implications of long transients in ecological systems. Nat Ecol Evol 5:285-294

Hastings A, Abbott KC, Cuddington K, Francis T, Gellner G, Lai Y-C, Morozov A, Petrovskii S, Scranton K, Zeeman ML (2018) Transient phenomena in ecology. Science 361:eaat6412

Hastings A, Abbott KC, Cuddington K, Francis TB, Lai Y-C, Morozov A, Petrovskii S, Zeeman ML (2021) Effects of stochasticity on the length and behavior of ecological transients. R Soc Interface 18:20210257

Morozov A, Abbott KC, Cuddington K, Francis T, Gellner G, Hastings A, Lai Y-C, Petrovskii S, Scranton K, Zeeman ML (2020) Long transients in ecology: theory and applications. Phys Life Rev 32:1-40

Reimer JR, Arroyo-Esquivel J, Jiang J, Scharf HR, Wolkovich EM, Zhu K, Boettiger C (2021) Noise can create or erase long transients. Theor Ecol this issue

Tao Y, Hite J, Lafferty KD, Earn DJD, Bharti N (2021) Transient disease dynamics across ecological scales. Theor Ecol this issue

Vidiella B, Fontich E, Valverde S, Sardanyés J (2021) Habitat loss causes long extinction transients in small trophic chains. Theor Ecol this issue 